\title{
$\left(\mathrm{Bi}_{0.85} \mathrm{Sb}_{0.15}\right)_{1-x} \mathrm{As}_{x}$ 的电输运和红外光学特性研究
}

\author{
蔡丽君 ${ }^{1}$, 史烜岱 ${ }^{1}$, 吴济穷 ${ }^{1}$, 朱圣云 ${ }^{1}$, 黄 耀 ${ }^{1}$, 侯延辉 ${ }^{1,2}$, 马永昌 ${ }^{1,3}$
}

(天津理工大学 1. 材料科学与工程学院; 2. 天津市光电显示材料与器件重点实验室; 3. 显示材料与光电器件省部 共建教育部重点实验室, 天津 300384)

摘 要: 采用熔融法制备了 $\left(\mathrm{Bi}_{0.85} \mathrm{Sb}_{0.15}\right)_{1-x} \mathrm{As}_{x}$ 合金, 用 $\mathrm{X}$ 射线衍射和电子能谱仪进行物相和组份表征, 随 $\mathrm{As}$ 掺杂量


电阻温度关系呈现半导体特性, 而 $\left(\mathrm{Bi}_{0.85} \mathrm{Sb}_{0.15}\right)_{0.95} \mathrm{As}_{0.05}$ 在 $12 \sim 300 \mathrm{~K}$ 范围都显示金属性。从红外反射光谱可知, $\mathrm{Bi}_{0.85} \mathrm{Sb}_{0.15}$ 的等离子边在远红外区且随温度下降向低频移动, 是窄带隙半导体的热激发行为。室温下 $\left(\mathrm{Bi}_{0.85} \mathrm{Sb}_{0.15}\right)_{0.95} \mathrm{As}_{0.05}$ 的自由电子等离子频率相比母体移动并不明显, 但是散射率增大, 在中红外 $600 \sim 2000 \mathrm{~cm}^{-1}$ 区 间光电导谱比 $\mathrm{Bi}_{0.85} \mathrm{Sb}_{0.15}$ 高, 经分析可知是源于带尾态的出现。综合对电输运和红外光谱的分析可知, $\left(\mathrm{Bi}_{0.85} \mathrm{Sb}_{0.15}\right)_{0.95} \mathrm{As}_{0.05}$ 的费米能级应处于扩展态区, 而并非定域态。

关 键 词: 红外光谱; Bi-Sb 合金; $\mathrm{As}$ 掺杂

中图分类号: O469; O43 文献标识码: A

\section{Electric Transport and Infrared Property of $\left(\mathrm{Bi}_{0.85} \mathrm{Sb}_{0.15}\right)_{1-x} \mathrm{As}_{x}$}

\author{
CAI Li-Jun ${ }^{1}$, SHI Xuan-Dai ${ }^{1}$, WU Ji-Qiong ${ }^{1}$, ZHU Sheng-Yun ${ }^{1}$, \\ HUANG Yao ${ }^{1}$, HOU Yan-Hui ${ }^{1,2}$, MA Yong-Chang ${ }^{1,3}$
}

(1. School of Materials Science and Engineering, Tianjin University of Technology, Tianjin 300384, China; 2. Tianjin Key Lab for Photoelectric Materials and Devices, Tianjin 300384, China; 3. Key Laboratory of Display Materials and Photoelectric Devices (Tianjin University of Technology), Ministry of Education, Tianjin 300384, China)

\begin{abstract}
Bi}_{85} \mathrm{Sb}_{15}\right)_{100-x} \mathrm{As}_{x}$ alloys were grown by melting stoichiometric mixture of elements $\mathrm{Bi}, \mathrm{Sb}$, and As. The phases and components of samples were analyzed by X-ray diffraction and the energy dispersion analysis. The As-doped (less than $8 \%$ in nominal) alloys have no impurity phases. Below $T=100 \mathrm{~K}, \mathrm{Bi}_{0.85} \mathrm{Sb}_{0.15}$ reveals semiconductor behavior in temperature dependent dc-resistivity, whereas $\left(\mathrm{Bi}_{0.85} \mathrm{Sb}_{0.15}\right)_{0.95} \mathrm{As}_{0.05}$ shows a metallic characteristic in the measured temperature range. The plasma of $\mathrm{Bi}_{0.85} \mathrm{Sb}_{0.15}$ shift towards low energy in far infrared reflectance spectra with the decrease of temperature, indicating thermally excited response of free electrons. Comparing with undoped $\mathrm{Bi}_{0.85} \mathrm{Sb}_{0.15}$, the plasma frequency of $5 \%$ As doped compound changes slightly, whereas the scattering rate of the free carriers increases. The infrared conductivity spectrum is enhanced in the range $600 \mathrm{~cm}^{-1}-2000 \mathrm{~cm}^{-1}$, due to the formation of tails of the energy bands. Combining with the dc electric transport and the analysis of infrared properties, the Fermi level of $\left(\mathrm{Bi}_{0.85} \mathrm{Sb}_{0.15}\right)_{0.95} \mathrm{As}_{0.05}$ possibly is not situated in the localized states, but in delocalized range.
\end{abstract}

Key words: infrared spectra; Bi-Sb alloys; As doping

收稿日期: 2017-10-17; 收到修改稿日期：2018-01-02

基金项目: 国家自然科学基金(10704054); 天津理工大学国家级大学生创新创业训练计划(201610060030)

National Natural Science Foundation of China (10704054); State Training Program of Innovation and Entrepreneurship of Tianjin University of Technology (201610060030)

作者简介: 蔡丽君(1992-), 男, 学士. E-mail: 18722155015@163.com

通讯作者: 马永昌, 副教授. E-mail: ycma@tjut.edu.cn 
掺杂半导体的电子输运特性是当前凝聚态物理 领域的重要研究方向之一。杂质浓度的增加将使材 料体系电子能带结构发生变化, 进而改变物理特 性。 $\mathrm{Bi}_{1-\alpha} \mathrm{Sb}_{\alpha}$ 合金从 1960 年代起即被认为是很有希 望的低温区热电材料 ${ }^{[1]}$, 之后很多工作是对 $\mathrm{Bi}_{1-\alpha} \mathrm{Sb}_{\alpha}$ 的制备工艺或超晶格的电子输运特性进行研究 ${ }^{[2-9]}$, 特别是近年来科学家发现 $\mathrm{Bi}_{1-\alpha} \mathrm{Sb}_{\alpha}$ 合金还是拓扑绝 缘体家族的重要成员, Singh 等 ${ }^{[10]}$ 通过第一性原理 计算预测了在高压下存在外尔 (Weyl) 半金属相, Sahin 和 Michael ${ }^{[11]}$ 在 $\mathrm{Bi}_{0.83} \mathrm{Sb}_{0.17}$ 中发现了巨自旋霍 尔电导效应, 由此可见 $\mathrm{BiSb}$ 合金电子输运特性和能 带结构是当前的研究热点 ${ }^{[12]}$ 。

已有实验结果表明, 随着 $\mathrm{Sb}$ 含量的增加,二元 $\mathrm{Bi}_{1-\alpha} \mathrm{Sb}_{\alpha}$ 合金的物理性质表现出 “半金属 $(\alpha<7 \%)$-半 导体 $(7 \%<\alpha<22 \%)$-半金属 $(\alpha>22 \%)$ " 的演化趋势 ${ }^{[6,8]}$ 。 对于 $\alpha<7 \%$ 的半金属区, $\mathrm{Bi}_{0.96} \mathrm{Sb}_{0.04}$ 电阻存在量子振 荡现象 ${ }^{[13]}$; 在 $\alpha>22 \%$ 的半金属区, $\mathrm{P}$ 型 $\mathrm{Sn}$ 掺杂的系 列样品的热电性能有明显的提高 ${ }^{[14]}$; 而对于半导体 区的材料, 人们研究了掺杂和改变制备工艺对低温 热电性能的影响 ${ }^{[4,9,15]}$ 。Benia 等 ${ }^{[2]}$ 近期研究了 $0<\alpha<0.6$ 范围的电子能带结构, 发现 $\mathrm{Bi}_{1-\alpha} \mathrm{Sb}_{\alpha}$ 合金表 面存在 2 个能带, 与理论计算相符合。根据 $\mathrm{Bi}-\mathrm{Sb}$ 合金相图可知 ${ }^{[5,8,14]}$, 由于 La、 Ls、H 和 $\mathrm{T}$ 能带结构 随 $\mathrm{Bi} / \mathrm{Sb}$ 比例而变化, $\mathrm{Bi}_{85} \mathrm{Sb}_{15}$ 在基态下禁带宽度可 以达到最大值 $30 \mathrm{meV}$ 。研究此组份的母体掺杂, 能 够相对容易观测电子能带结构的变化, 同时可加深 理解掺杂对 $\mathrm{Bi}-\mathrm{Sb}$ 合金载流子输运特性的影响。因 $\mathrm{Bi} 、 \mathrm{Sb} 、 \mathrm{As}$ 能在很宽的比例范围内固溶 ${ }^{[16]}$, 且 $\mathrm{As}$ 具有与 $\mathrm{Bi} 、 \mathrm{Sb}$ 相似的电子构型, 所以对 $\mathrm{Bi}_{0.85} \mathrm{Sb}_{0.15}$ 进行 As 掺杂并不引入多余载流子而仅使体系的无 序程度增加, 所研究的问题也相应简化。如果费米 能级处于因无序导致的定域态区时，体系基态下的 电导率为零, 呈现非金属性; 而如果费米能级处于 扩展态区时，体系将会呈现金属性的电导 ${ }^{[17]}$; 那么 对于基态下带隙只有 $30 \mathrm{meV}$ 的 $\mathrm{Bi}_{0.85} \mathrm{Sb}_{0.15}$, 掺杂 $\mathrm{As}$ 后其载流子在低温下是否为定域行为? 体系能带结 构怎样改变? 这些问题都尚待探索。

红外光谱是研究诸多固体电子能带结构的强有 力工具之一 ${ }^{[18-22]}$, 能量分辨率高达 $0.1 \mathrm{meV}$, 可以精 确地探测到窄带隙半导体材料在费米能级附近较高 能量电子的信息, 而这些电子正是直流电输运特性 的主要贡献者, 所以研究红外光学特性有助于分析 材料的电学本质属性。As 掺杂 $\mathrm{Bi}-\mathrm{Sb}$ 合金的电输运 性质和红外光谱目前尚无报导; 即使对未掺杂的 $\mathrm{Bi}_{0.85} \mathrm{Sb}_{0.15}$, 电子能态和载流子散射率的数据也多 数来自直流电输运而并非红外光谱 ${ }^{[5-8]}$ 。本研究组近
期生长了系列 $\left(\mathrm{Bi}_{0.85} \mathrm{Sb}_{0.15}\right)_{1.0-x} \mathrm{As}_{x}$ 晶体并测量了直流 电输运和红外光学特性。

\section{1 实验方法}

按照名义配比 $\left(\mathrm{Bi}_{0.85} \mathrm{Sb}_{0.15}\right)_{1.0-x} \mathrm{As}_{x}(x=0,5 \%, 8 \%$, $10 \%$ )称量所需的高纯度(其中 $\mathrm{Bi}$ 和 $\mathrm{Sb}$ 为光学纯, $\mathrm{As}$ 不低于 $99.99 \%$ )单质化学试剂, 装入石英管中, 抽 真空氢氧焰封接后在箱式炉中加热至 $700^{\circ} \mathrm{C}$ 开始恒 温, 为了确保反应充分, 恒温过程中每隔 $1 \mathrm{~h}$ 进行一 次翻转, $20 \mathrm{~h}$ 后停止翻转。然后以 $3^{\circ} \mathrm{C} / \mathrm{h}$ 的速度缓慢 冷却至 $200^{\circ} \mathrm{C}$, 最后自然冷却到室温, 得到系列晶 体试样。

采用 DMax-2500 型 X 射线衍射仪 ( $\mathrm{Cu}-\mathrm{K} \alpha$ 射线) 对样品进行物相分析, 通过 SEM 和能谱扫描观测 样品成份和微观形貌。用 7265 DSP 锁相放大器(频 率 $120 \mathrm{~Hz}$, 四探针)测量电阻率随温度 $(12 \sim 300 \mathrm{~K})$ 变 化曲线, 低温控温在闭循环变温恒温器 (Janis) 和 Cryo con32 控温仪器上实现, 控温精度小于 $0.01 \mathrm{~K}$ 。 为了获得样品电子能带结构信息, 采用傅里叶红外 光谱仪 Bruker $80 \mathrm{v} / \mathrm{S}$ 在 $10^{-5} \mathrm{~Pa}$ 的高真空下结合原位 蒸镀金膜技术, 对表面尺寸为 $3 \mathrm{~mm} \times 3 \mathrm{~mm}$ 平整光 亮的样品先进行反射率谱 $R(\omega)$ (近垂直入射, 30 $15000 \mathrm{~cm}^{-1}$ 测试, 其中远红外(30 700 $\left.\mathrm{cm}^{-1}\right)$ 探测器 需要液氦冷却, 再通过以下步骤得到光电导率谱 $\left.\sigma_{1}(\omega)^{[21-22]}: 1\right)$ 由 Kramers-Kronig $(\mathrm{K}-\mathrm{K})$ 变换计算反 射光相位改变, $\theta(\omega)=-\frac{\pi}{\omega} P \int_{0}^{\infty} \frac{\ln R(s)}{s^{2}-\omega^{2}} \mathrm{~d} s$, 其中 $P$ 表 示主值积分，积分的低频(下限)部分外推采用 Hagen- Rubens 关系, 即 $R(\omega)=1-2 \sqrt{2 \varepsilon_{0} \omega / \sigma_{0}}\left(\varepsilon_{0}\right.$ 是 真空介电常数, $\sigma_{0}$ 是直流电导率), 而高频(上限) 部 分外推采取 $R(\omega) \sim \omega^{-2}$ 形式;2) 计算出反射系数 $r=$ $R^{1 / 2} e^{\mathrm{i} \theta}=(n-1+i k) /(n+1+i k)$ 和复折射率 $N=n+i k=\varepsilon^{1 / 2}$ (复 介电常数 $\left.\varepsilon=\varepsilon_{1}+i \varepsilon_{2}\right) ; 3$ ) 根据复电导率和复介电常数 关系可得, $\sigma_{1}(\omega)=\omega \varepsilon_{2}(\omega) / 4 \pi$ 。

\section{2 结果与讨论}

掺杂 As 含量为 $0,5 \%, 8 \%, 10 \%$ 的样品(晶体研 磨成粉末后)XRD 图谱如图 1 所示, 衍射峰与粉末 衍射数据库标准卡片 PDF\#44-1246 的相一致, 结构 对应 $\mathrm{R} 3 \mathrm{~m}$ 空间群。名义掺杂 $5 \%$ 以下的样品为单相 合金，对于 $x=8 \%$ 的样品开始出现少量杂相，而 $x=10 \%$ 的样品杂相峰明显, 如图 1 中的*所示。XRD 低角度峰分析结果和 As 元素比例如表 1 所示, 随着 掺杂 As 浓度的增加, 晶胞 $a$ 轴和 $c$ 轴的尺寸均减小, 


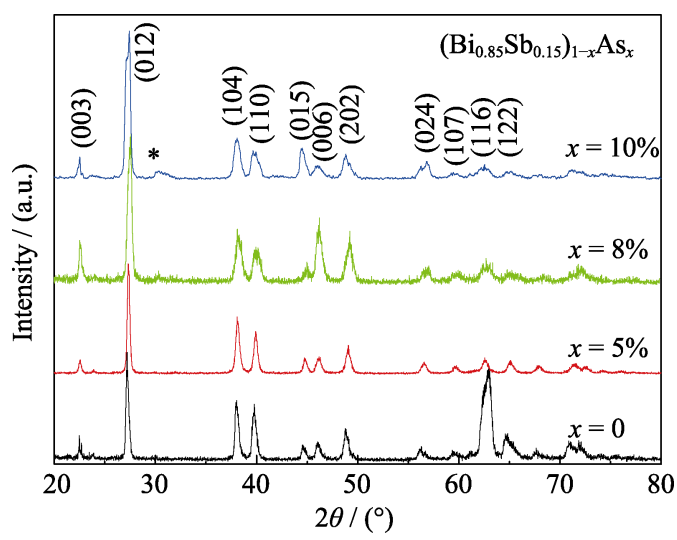

图 $1 \quad\left(\mathrm{Bi}_{0.85} \mathrm{Sb}_{0.15}\right)_{1.0-x} \mathrm{As}_{x}$ 合金的 XRD 图谱

Fig. 1 XRD patterns of $\left(\mathrm{Bi}_{0.85} \mathrm{Sb}_{0.15}\right)_{1.0-x} \mathrm{As}_{x}$ alloys

表 1 样品中 As 元素含量和晶格尺寸

Table 1 Content of As in samples and lattice sizes

\begin{tabular}{cccc}
\hline $\begin{array}{c}\text { Nominal } \\
\text { content As/\% }\end{array}$ & $\begin{array}{c}\text { Measured } \\
\text { content As/\% }\end{array}$ & $\begin{array}{c}\text { Crystal lattice } \\
a / \mathrm{nm}\end{array}$ & $\begin{array}{c}\text { Crystal lattice } \\
c / \mathrm{nm}\end{array}$ \\
\hline 0 & 0 & 0.4552 & 1.1913 \\
5 & 2.5 & 0.4528 & 1.1842 \\
8 & 3.8 & 0.4487 & 1.1811 \\
\hline
\end{tabular}

是因 $\mathrm{As}$ 比 $\mathrm{Bi}$ 和 $\mathrm{Sb}$ 原子半径小, 从而导致晶格收缩。

图 2(a) (c) 是 $\left(\mathrm{Bi}_{0.85} \mathrm{Sb}_{0.15}\right)_{0.95} \mathrm{As}_{0.05}$ 合金中各元 素的分布图, 从图中可以看出, As 确实固溶进了 $\mathrm{Bi}-\mathrm{Sb}$ 合金。未掺杂的晶体样品 $\mathrm{Bi}_{0.85} \mathrm{Sb}_{0.15}$ 解理性良
好(图 2(d)), 而 5\% As 样品的解理性能变差(图 2(e))。 样品成份测量结果(微区面积为 $20 \mu \mathrm{m} \times 20 \mu \mathrm{m}$ ) 显示 实际 As 浓度要小于名义组份, 是由于易挥发的 As 沉积在石英管壁或者合金块(锭)的表面。

由样品电阻率-温度曲线(图 3)可以看出, 未掺 杂的 $\mathrm{Bi}_{0.85} \mathrm{Sb}_{0.15}$ 母体以及掺杂 $5 \% 、 8 \% \mathrm{As}$ 后的样品 电阻率在 $120 \mathrm{~K}$ 以上随着温度升高而增加, 说明体 系有大量的自由电子存在, 温度升高之后晶格振动 散射增加使电阻率加大 ${ }^{[6,23]}$ 。在 $300 \mathrm{~K}$ 三个样品的 直流电阻率都大约为 $0.2 \mathrm{~m} \Omega \cdot \mathrm{cm}$ 。Ravindra 等 ${ }^{[24]}$ 和 Vurgaftman 等 ${ }^{[25]}$ 的研究指出, Bi-Sb 合金的带隙与温 度有关，这是由于温度变化导致电-声子相互作用 或者晶格间距改变造成的。未掺杂合金的电阻率在 $100 \mathrm{~K}$ 以下轻微上升, 对应半导体带隙的逐渐打 开 ${ }^{[6,24]}$, 而对于 $50 \mathrm{~K}$ 以下电阻对温度的依赖关系, 主要反映了载流子的迁移率随温度的变化行为 ${ }^{[6]}$ 。 对于掺杂 $5 \% \mathrm{As}$ 的样品 $\left(\mathrm{Bi}_{0.85} \mathrm{Sb}_{0.15}\right)_{0.95} \mathrm{As}_{0.05}$, 整体 测试温区呈现金属性，低温下的剩余电阻率约为 $0.08 \mathrm{~m} \Omega \cdot \mathrm{cm}$, 根据马西森定则可知其起源于样品 内的杂质和成份不均匀 ${ }^{[23]}$ 。相比未掺杂的母体 $\mathrm{Bi}_{0.85} \mathrm{Sb}_{0.15}$, 半导体特征消失。掺杂 $8 \% \mathrm{As}$ 的样品电 阻率随温度下降先减小后(在 $100 \mathrm{~K}$ 以下)一直增加, 显然, 在 $50 \mathrm{~K}$ 以下的电阻率变化趋势与其他两个样 品不同。
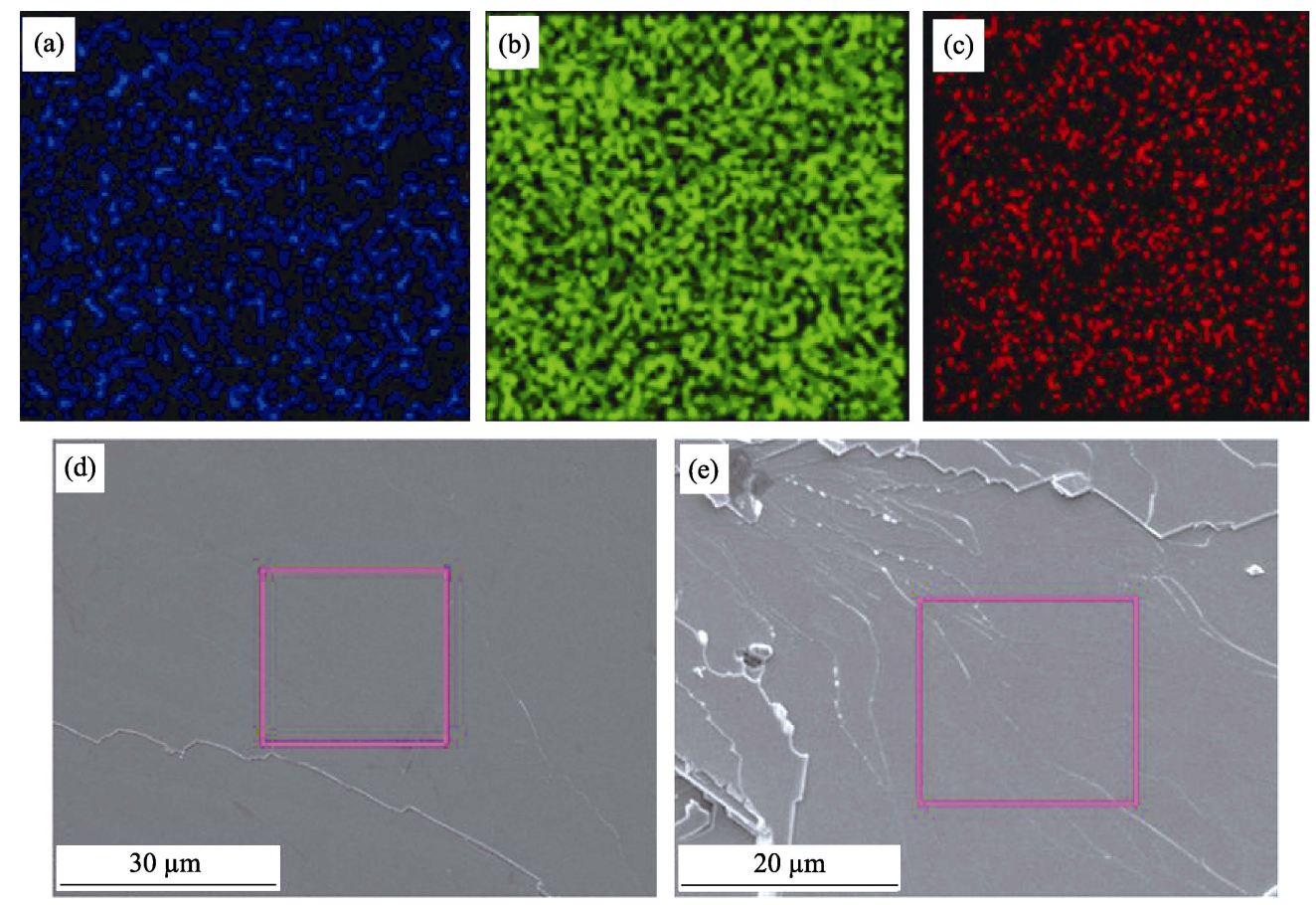

图 $2 \mathrm{Sb}$ 元素(a)、Bi 元素(b)和 $\mathrm{As}$ 元素(c)在 $\left(\mathrm{Bi}_{0.85} \mathrm{Sb}_{0.15}\right)_{0.95} \mathrm{As}_{0.05}$ 合金中实空间 $(20 \mu \mathrm{m} \times 20 \mu \mathrm{m})$ 的分布图;

未掺杂 $\mathrm{Bi}_{0.85} \mathrm{Sb}_{0.15}$ 样品 $(\mathrm{d})$ 和 $\left(\mathrm{Bi}_{0.85} \mathrm{Sb}_{0.15}\right)_{0.95} \mathrm{As}_{0.05}$ 样品(e)解理后的微观形貌，其中的矩形图框为能谱扫描范围

Fig. 2 Elements mapping of $\mathrm{Sb}(\mathrm{a}), \mathrm{Bi}(\mathrm{b})$ and $\mathrm{As}(\mathrm{c})$ in a range of $20 \mu \mathrm{m} \times 20 \mu \mathrm{m}$ in $\left(\mathrm{Bi}_{0.85} \mathrm{Sb}_{0.15}\right)_{0.95} \mathrm{As}_{0.05}$, microphologies of the cleaved surface of undoped $\mathrm{Bi}_{0.85} \mathrm{Sb}_{0.15}(\mathrm{~d})$ and $\left(\mathrm{Bi}_{0.85} \mathrm{Sb}_{0.15}\right)_{0.95} \mathrm{As}_{0.05}$ sample (e)

The rectangles present the scanning area for $\left(\mathrm{Bi}_{0.85} \mathrm{Sb}_{0.15}\right)_{0.95} \mathrm{As}_{0.05}$ and undoped $\mathrm{Bi}_{0.85} \mathrm{Sb}_{0.15}$, respectively 


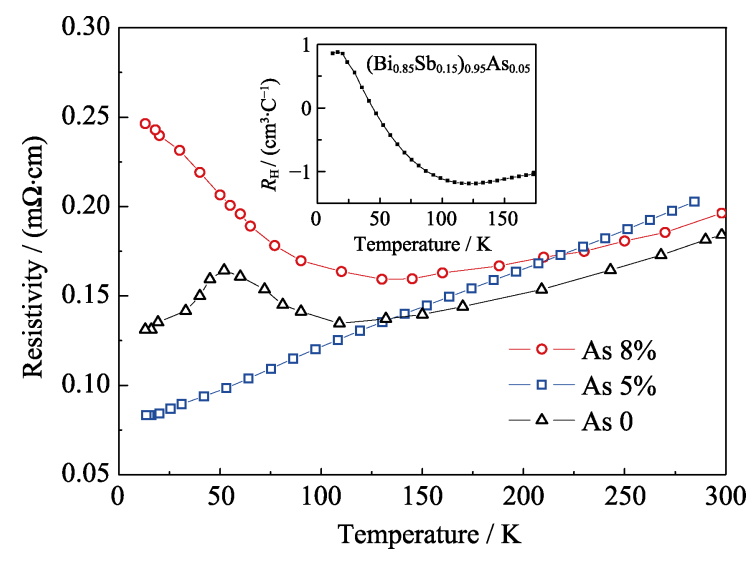

图 3 不同掺杂浓度 $\left(\mathrm{Bi}_{0.85} \mathrm{Sb}_{0.15}\right)_{1.0-x} \mathrm{As}_{x}(x=0,5 \%, 8 \%)$ 电阻 率-温度曲线

Fig. 3 Temperature dependent resistivity of $\left(\mathrm{Bi}_{0.85} \mathrm{Sb}_{0.15}\right)_{1.0-x} \mathrm{As}_{x}$ $(x=0,5 \%, 8 \%)$

Inset shows the temperature dependence of Hall coefficient

为了能够对电输运特性进行深入分析, 测量并 比较了掺杂 $5 \% \mathrm{As}$ 和未掺杂样品 $\mathrm{Bi}_{0.85} \mathrm{Sb}_{0.15}$ 的红外 光谱。从图 4 变温红外反射率谱可知, 未掺杂母体 $\mathrm{Bi}_{0.85} \mathrm{Sb}_{0.15}$ 室温下的反射率在小于 $200 \mathrm{~cm}^{-1}$ 的低频 区高于 0.95 , 超过 $200 \mathrm{~cm}^{-1}$ 以后快速下降, 是自由 电子的响应 ${ }^{[21]}$ 。随着温度下降, 红外区的等离子边 $\omega_{\mathrm{p}}$ 从 $300 \mathrm{~K}$ 时大约为 $300 \mathrm{~cm}^{-1}$ 向低频移动(如图中 箭号所示), 远红外区的反射率被压制, 即自由电子 响应变弱。由于载流子浓度 $n \propto \omega_{\mathrm{p}}^{2[21]}$, 所以温度降 低载流子浓度下降, 对应半导体热激发载流子的行 为 $^{[18]}$ 。这里可以解释实验中观测到的(图 3) $120 \mathrm{~K}$ 以 上 $\mathrm{Bi}_{0.85} \mathrm{Sb}_{0.15}$ 的电阻率 - 温度关系: 由于材料的电阻 率同时受自由载流子浓度和散射率的影响, 而载流 子的散射率随温度下降应该减小 ${ }^{[21,23]}$, 也即迁移率 随温度下降而增加, 所以在 $120 \mathrm{~K}$ 以上 $\mathrm{Bi}_{0.85} \mathrm{Sb}_{0.15}$ 的电阻率随着温度升高而增加。

从图 5 中可以看出, 掺杂 $5 \% \mathrm{As}$ 与未掺杂 $\mathrm{As}$ 样

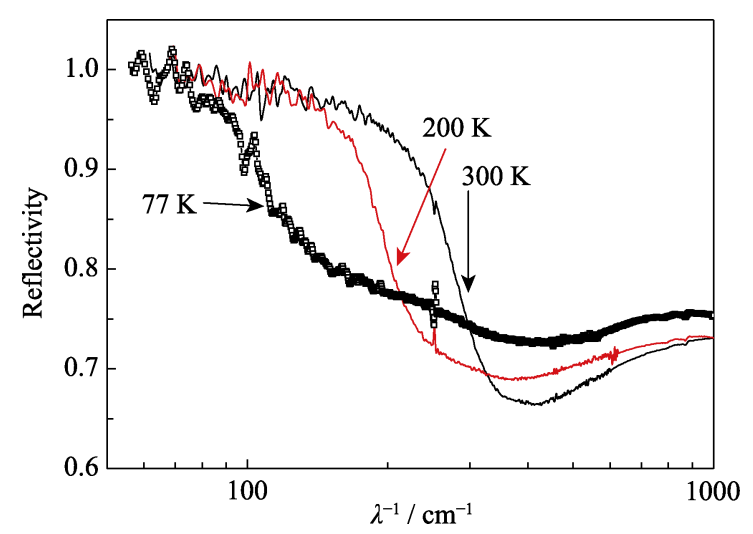

图 $4 \mathrm{Bi}_{0.85} \mathrm{Sb}_{0.15}$ 的变温红外反射率谱

Fig. 4 Infrared reflectivity of $\mathrm{Bi}_{0.85} \mathrm{Sb}_{0.15}$ at various temperatures The arrows represent positions of the plasma edges

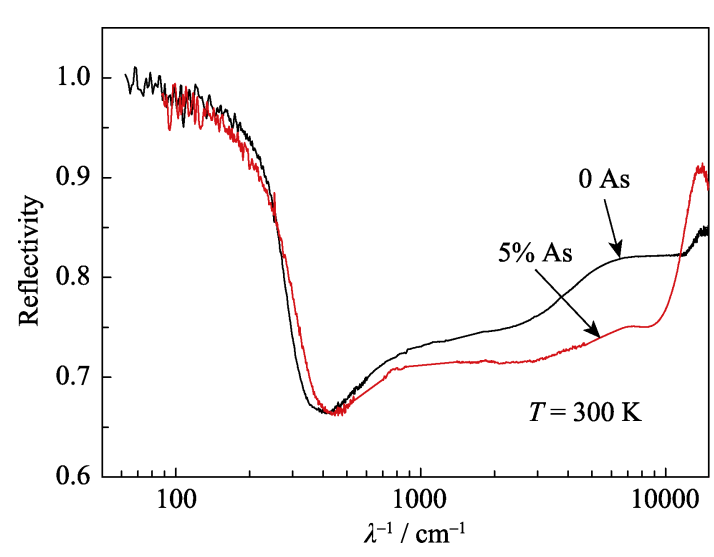

图 5 掺杂 $5 \% \mathrm{As}$ 的样品与未掺杂 $\mathrm{As}$ 样品在 $300 \mathrm{~K}$ 下的红 外反射率

Fig. 5 Infrared reflectivity of the undoped $\mathrm{Bi}_{0.85} \mathrm{Sb}_{0.15}$ and $\left(\mathrm{Bi}_{0.85} \mathrm{Sb}_{0.15}\right)_{0.95} \mathrm{As}_{0.05}$ at $300 \mathrm{~K}$

品在远红外区 $200 \mathrm{~cm}^{-1}$ 以下反射率均较大, 对应较 高的直流电导率 ${ }^{[21-22]}$ 。掺杂 As 之后的等离子振荡 频率与未掺杂情况相比非常接近, 均在 $300 \mathrm{~cm}^{-1}$ 附 近, 说明掺杂 As 之后载流子浓度变化不明显, 符合 预期。考虑到室温下掺杂 $5 \%$ As 样品的直流电阻率 高, 表明载流子的散射率在掺杂后加大。在中红外 800 2000 $\mathrm{cm}^{-1}$ 和近红外 3500 12000 $\mathrm{cm}^{-1}$ 区间二者 的反射率谱中都出现了带间跃迁的特征 ${ }^{[18]}$ 。

从计算得到的光电导谱(图 6)可见，在 $600 \mathrm{~cm}^{-1}$ 以下的远红外区红外光电导随频率降低而升高的行 为是自由电子的特征 ${ }^{222]}$ 。考虑到低频光电导数据是 掺杂样品稍高, 而 $300 \mathrm{~K}$ 下直流电导率(对应光电导 率向低频区外推的极限) 是未掺杂母体更高, 所以 只能由 As 掺杂后载流子散射率增加才可解释, 也 就是说两者的光电导谱曲线在更低能量处应有交 叉。结合直流电阻-温度关系推断费米能级处于扩展 态区, 而不是在定域态: 因为如果费米能级位于定

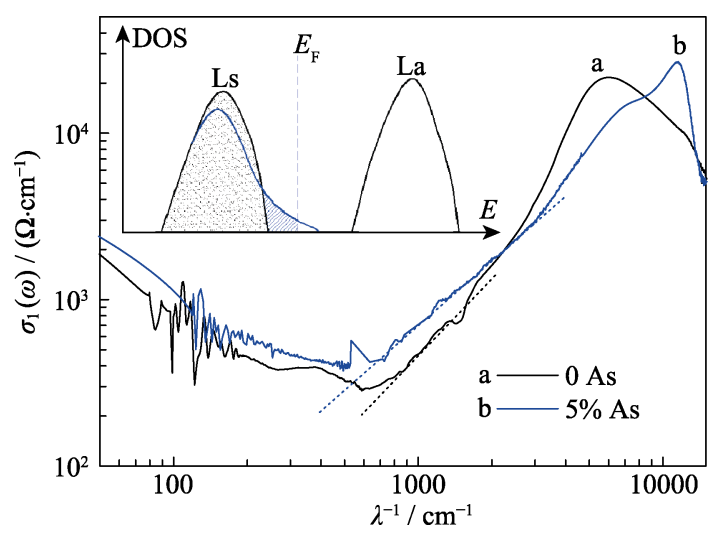

图 6 掺杂 $5 \% \mathrm{As}$ 与未掺 $\mathrm{As}$ 样品在 $300 \mathrm{~K}$ 下的红外光电导谱 Fig. 6 Infrared conductivity spectra of the undoped $\mathrm{Bi}_{0.85} \mathrm{Sb}_{0.15}$ and $\left(\mathrm{Bi}_{0.85} \mathrm{Sb}_{0.15}\right)_{0.95} \mathrm{As}_{0.05}$ at $300 \mathrm{~K}$

Inset is the proposed diagram of energy bands structure (density of states, DOS) under ground state near the Fermi level 
域态区 ${ }^{[17]}$, 则低温下直流电阻率应该是非金属行为, 而实验数据给出直到 $T=12 \mathrm{~K}$ 时, 直流电阻率也未 随温度降低而上升, 因此可以断定在本实验温区范 围内 $\left(\mathrm{Bi}_{0.85} \mathrm{Sb}_{0.15}\right)_{0.95} \mathrm{As}_{0.05}$ 的载流子并未被定域化。 $12 \mathrm{~K}$ 温度时, 激发能量大约相当于 $1.0 \mathrm{meV}$, 所以可 以判断即便掺杂和缺陷等无序能够引起电子定域, 那么对应的定域化能量也会小于 $1.0 \mathrm{meV}$ 。为了能够 进一步研究 $\left(\mathrm{Bi}_{0.85} \mathrm{Sb}_{0.15}\right)_{0.95} \mathrm{As}_{0.05}$ 的电输运特性, 测量 了 Hall 系数以估算材料体系的载流子浓度, 如图 3 的插图所示。然而实验结果显示在 $40 \mathrm{~K}$ 附近, $\left(\mathrm{Bi}_{0.85} \mathrm{Sb}_{0.15}\right)_{0.95} \mathrm{As}_{0.05}$ 的 Hall 系数出现了正负号的转 变, 无法直接用单带模型准确计算体系的载流子 浓度。在 $T=40 \mathrm{~K}$ 以下, Hall 系数为正, 可以判定 $\left(\mathrm{Bi}_{0.85} \mathrm{Sb}_{0.15}\right)_{0.95} \mathrm{As}_{0.05}$ 的导电贡献主要来自于空穴型 载流子。

中红外区光电导谱反映出带间跃迁特征 ${ }^{[18]}$, 如 图 6 虚线所示: 光电导率在 $600 \mathrm{~cm}^{-1}$ 附近开始随波 数增加而增大, 对应 $\mathrm{Ls}$ 到 $\mathrm{La}$ 能带的跃迁 ${ }^{[9,24]}$ 。我们 注意到在 600 2000 $\mathrm{cm}^{-1}$ 范围内掺杂 $5 \%$ As 样品的 光电导率高, 既然红外光电导谱探测电子从占据态 到空态的跃迁 ${ }^{[21]}$, 而从前面分析得出体系中并不存 在价带和导带之间的定域态, 并且电子浓度也未因 掺杂改变, 那么这个光电导的增加应该是起源于占 据态电子态密度发生的变化, 所以合理解释是带尾 态的出现(如图 6 插图所示, 阴影区为占据态)。对于 无序系统的电子能态, 经常在带顶和带底出现带尾 态分布 ${ }^{[17]}$ 。就 $\left(\mathrm{Bi}_{0.85} \mathrm{Sb}_{0.15}\right)_{1.0-x} \mathrm{As}_{x}$ 体系来说, 无序的 贡献者应当包含 As 原子的随机分布、合金体系的 成份不均匀和晶格缺陷等。

从 2500 到 $15000 \mathrm{~cm}^{-1}$ 的高能波段, 还有两个光 电导率的峰值出现, 由能量间隔更大的带间跃迁所 贡献, 显然 As 掺杂对电子在能带间的跃迁有影响: 在 $8000 \mathrm{~cm}^{-1}$ 以下光电导被压制, 同时峰值向高能 方向移动, 表明电子的束缚能有所加强, 但是这部 分被强烈束缚的电子对样品电输运特性的影响可以 忽略，在此不作深入讨论。

\section{3 结论}

采用熔融法制备了系列 $\left(\mathrm{Bi}_{0.85} \mathrm{Sb}_{0.15}\right)_{1.0-x} \mathrm{As}_{x}$ 样 品。 $\mathrm{X}$ 射线物相分析和能谱测试结果表明, 掺杂低 浓度 $\mathrm{As}$ 的 $\mathrm{Bi}-\mathrm{Sb}$ 合金为单相，掺杂后晶格收缩。对 于未掺杂的母体 $\mathrm{Bi}_{0.85} \mathrm{Sb}_{0.15}$, 随温度下降红外区的 等离子边向低频移动, 是窄带半导体的热激发行 为。掺杂 5\% As 的样品, 显示了近似金属性的直流 电阻温度关系。从对样品的红外光谱分析可知, 掺
杂 $5 \% \mathrm{As}$ 样品的等离子频率与 $\mathrm{Bi}_{0.85} \mathrm{Sb}_{0.15}$ 相比移动 并不明显, 说明载流子浓度几乎没有改变, 但是载 流子的散射率在掺杂 As 后增大。As 掺杂可以改变 $\mathrm{Bi}_{0.85} \mathrm{Sb}_{0.15}$ 合金的能带结构，出现因无序导致的带 尾态密度分布。结合直流电输运实验的测量结果, 可判断 $\left(\mathrm{Bi}_{0.85} \mathrm{Sb}_{0.15}\right)_{0.95} \mathrm{As}_{0.05}$ 的费米能级在扩展态区, 而处于非定域态区。

致谢: 感谢北京大学物理学院固体光谱实验室课题 组在反射率谱测量方面的大力支持。

\section{参考文献:}

[1] SNYDER G J, TOBERER E S. Complex thermoelectric materials. Nature Materials, 2008, 7(2): 105-114.

[2] BENIA H, STRABER C, KERN K, et al. Surface band structure of $\mathrm{Bi}_{1-x} \mathrm{Sb}_{x}(111)$. Phys. Rev. B, 2015, 91(16): 161406(R)-1-5.

[3] NOGUCHI H, KITAGAWA H, KIYABU T, et al. Low temperature thermoelectric properties of Pb- or Sn-doped Bi-Sb alloys. J. Phys. Chem. Solids, 2007, 68(1): 91-95.

[4] LIU H J, SONG C M, WU S T, et al. Processing method dependency of thermoelectric properties of $\mathrm{Bi}_{85} \mathrm{Sb}_{15}$ alloys in low temperature. Cryogenics, 2007, 47(1): 56-60.

[5] SUNGLAE C, YUNKI K, SUK Y, et al. Artificially ordered Bi/Sb superlatitice alloys: fabrication and transport properties. Phys. Rev. B, 2001, 64(23): 235330-1-4.

[6] LENOIR B, CASSART M, MICHENAUD J P, et al. Transport properties of Bi-rich Bi-Sb alloys. J. Phys. Chem. Solids, 1996, 57(1): 89-99.

[7] CHEN Z, ZHOU M, HUANG R J, et al. Thermoelectric properties of p-type $\mathrm{Pb}$-doped $\mathrm{Bi}_{85} \mathrm{Sb}_{15-x} \mathrm{~Pb}_{x}$ alloys at cryogenic temperatures. J. Alloys Compd., 2012, 511(1): 85-89.

[8] ROGACHEVA E I, DROZDOVA A A, NASHCHEKINA O N, et al. Transition into a gapless state and concentration anomalies in the properties of $\mathrm{Bi}_{100-x} \mathrm{Sb}_{x}$ solid solutions. Appl. Phys. Lett., 2009, 94(20): 202111-1-4.

[9] DUTTA S, SHUBHA V, RAMESH T G. Effect of pressure and temperature on thermopower of Bi-Sb alloys. Physica B, 2010, 405(5): 1239-1243.

[10] SINGH S, GARCIA-CASTRO A C, IRAIS V, et al. Prediction and control of spin polarization in a Weyl semimetallic phase of BiSb. Phys. Rev. B, 2016, 94(16): 161116(R)-1-5.

[11] ŞAHIN CÜNEYT, MICHAEL E FLATTÉ. Tunable giant spin hall conductivities in a strong spin-orbit semimetal: $\mathrm{Bi}_{1-x} \mathrm{Sb}_{x}$. Phys. Rev. Lett., 2015, 114(10): 107201-1-5.

[12] ZHANG H J, LIU C X, QI X L, et al. Electronic structures and surface states of the topological insulator $\mathrm{Bi}_{1-x} \mathrm{Sb}_{x}$. Phys. Rev. B, 2009, 80(8): 085307-1-8.

[13] BANERJEE A, FAUQUE B, IZAWA K, et al. Transport anomalies 
across the quantum limit in semi-metallic $\mathrm{Bi}_{0.96} \mathrm{Sb}_{0.04}$. Phys. Rev. B, 2008, 78(16): 161103-1-4.

[14] HYUNGYU J, CHRISTOPHER M J, HEREMANS J P. Enhancement in the figure of merit of p-type $\mathrm{Bi}_{100-x} \mathrm{Sb}_{x}$ alloys through multiple valence-band doping. Appl. Phys. Lett., 2012, 101(5): 053904-1-5.

[15] LUO T T, WANG S Y, LI H, et al. Low temperature thermoelectric properties of melt spun $\mathrm{Bi}_{85} \mathrm{Sb}_{15}$ alloys. Intermetallics, 2013, 32: 96-102.

[16] TSUCHIYA Y. The molar volume of molten As-Sb, As-Bi and As-Te systems: further evidence for rapid structural changes in liquid As in the supercooled state. J. Non-Crystalline Sol., 1999, 250(2): 473-477.

[17] 阎守胜. 固体物理基础. 北京: 北京大学出版社, 2000: 283-290.

[18] DRESSEL M, GRUNER G. Electrodynamics of Solids: Optical Properties of Electrons in Matter. Beijing: Beijing World Publishing Corporation, 2005: 339-360.

[19] DEAN N, PETERSEN C, FAUSTI D, et al. Polaronic conductivity in the photoinduced phase of $1 \mathrm{~T}-\mathrm{TaS}_{2}$. Phys. Rev. Lett., 2011, 106(1): 016401-1-4.

[20] CHEN Z G, YUAN R H, DONG T, et al. Infrared spectrum and its implications for the electronic structure of the semiconducting iron selenide $\mathrm{K}_{0.83} \mathrm{Fe}_{1.53} \mathrm{Se}_{2}$. Phys. Rev. B, 2011, 83(22): 220507(R)-1-4.

[21] BASOV D N, TIMUSK T. Electrodynamics of high- $T_{\mathrm{c}}$ superconductors. Rev. Mod. Phys., 2005, 77(2): 721-778.

[22] HU B F, CHENG B, YUAN R H, et al. Coexistence and competition of multiple charge-density-wave orders in rare-earth tritellurides. Phys. Rev. B, 2014, 90(8): 085105-1-7.

[23] 陆 栋, 蒋 平. 固体物理学. 北京: 高等教育出版社, 2011: 104114.

[24] RAVINDRA N M, SRIVASTAVA V K. Temperature dependence of the energy gap in Bi-Sb systems. J. Phys. Chem. Solids, 1980, 41(11): $1289-1290$.

[25] VURGAFTMAN I, MEYER J R, RAM-MOHAN L R. Band parameters for III-V compound semiconductors and their alloys. $J$. Appl. Phys., 2001, 89(11): 5815-5875. 